\title{
Synthesis of Poly(ether)s with Pendant Ester Groups by the Polyaddition of Bis(oxetane)s with Active Bis(ester)s
}

\author{
Tadatomi Nishikubo, ${ }^{\dagger}$ Hiroto Kudo, Masaki Sasaki, Kenji Kuwamura, and Chiga ImataKi \\ Department of Applied Chemistry, Faculty of Engineering, Kanagawa University, \\ Rokkakubashi, Kanagawa-ku, Yokohama 221-8686, Japan
}

(Received May 18, 2004; Accepted June 30, 2004; Published October 15, 2004)

\begin{abstract}
Polyadditions of certain bis(oxetane)s such as 1,4-bis[(3-ethyl-3-oxetanylmethoxymethyl)]benzene (BEOB), 1,4-bis[(3-ethy-3-oxetanyl)methoxy]benzene (1,4-BEOMB), 4,4'-bis[(3-ethyl-3-oxetanyl)methoxy]benzene (4,4'-BEOBP), 2,2'-bis[(3-ethyl-3-oxetanyl)methoxy]biphenyl (2,2'-BEOBP), 3,3',5,5'-tetramethyl-[4,4'-bis(3-ethyl-3oxetanyl)methoxy]biphenyl (TM-BEOBP) with such active bis(ester)s as 4,4'-isopropylidenebis(2,6-dichlorophenyl diacetate) (IBDPA), 4,4'-isopropylidene-bis(2,6-dichlorophenyl dibenzoate) (IBDPB) and 4,4'-isopropylidenebis(2,6-dichlorophenyl di-2,4-dichlorobenzate) (IBDPD) were examined in the presence of tetraphenylphosphonium chloride (TPPC) as a catalyst in DMAc at $160^{\circ} \mathrm{C}$ for $96 \mathrm{~h}$, and it was found that corresponding polyethers with $M_{\mathrm{n}} \mathrm{s}$ in the range $7200-17400$ were obtained in $91-99 \%$ yields. The all obtained polymers would soluble in common organic solvents. [DOI 10.1295/polymj.36.841]

KEY WORDS Polyaddition / Bis(oxetane) / Active Bis(ester) / Synthesis of Poly(ether) / Pendant Ester Group / Catalyst /
\end{abstract}

It is well known that epoxy compounds have high chemical reactivity to many organic reagents ${ }^{1-4}$ such as amines, phenols, thiols, carboxylic acids, acyl halides, aryl esters, and thioesters, carbon dioxide as well as cationic catalysts ${ }^{5-7}$ and anionic catalysts ${ }^{8-10}$ due to the high strain energy. Therefore, epoxy resins and polymers with pendant epoxide groups have been used $^{1,2}$ as starting materials for the synthesis of functional polymers by the addition reaction of the epoxide groups with carboxylic acids, phenols, or amines. The above reaction systems of the epoxy resins and the polymers containing epoxide groups have also been applied as an important key technology in such industries as coatings, paintings, elastomers, adhesions, and electronics.

Meanwhile, oxetanes as 4-membered cyclic ether have the mostly same strain energy ${ }^{11}(107 \mathrm{~kJ} / \mathrm{mol})$ as oxiranes. However, we could not found useful reactions of oxetanes in the synthetic organic chemistry or in the polymer synthesis excepting old cationic ringopening polymerization, ${ }^{12}$ and of recent cationic isomerization by Motoi and co-workers. ${ }^{13-15}$

Therefore, the authors have been of great interest to develop new reactions of oxetanes, and found certain new addition reactions of oxetanes with carboxylic acids,${ }^{16}$ phenols, ${ }^{17,18}$ and thiophenols, ${ }^{19}$ using quaternary onium salts or crown ether complexes as catalysts. Since these addition reactions proceed smoothly without any side reactions even at higher temperatures than those of the reaction of oxiranes, the reactions can be used as suitable reaction for the polymer syn- thesis. More recently, our research group found anionic ring-opening alternating copolymerization ${ }^{20}$ of oxetane with carboxylic anhydride, and anionic ringopening polymerization ${ }^{21-23}$ of 3-ethyl-3-hydroxymethyloxetane. However, we do not have development of the addition reaction of oxetanes with phenyl esters until now, although we have found the addition reactions of oxetane with acyl chloride ${ }^{15-24}$ or $S$-aryl thioesters. ${ }^{26,27}$

From these backgrounds, in this article, we examined the polyaddition of bis(oxetane)s with active bis(ester)s, which were prepared by the reaction of bi$\mathrm{s}(\mathrm{phenol}) \mathrm{s}$ with carboxylic acid derivatives, for the synthesis of poly(ether)s containing pendant ester groups under appropriate reaction conditions.

\section{EXPERIMENTAL}

\section{Materials}

The reaction solvents, such as $N$-methylpyrrolidone (NMP), dimethylformamide (DMF), methylethylketone (MEK), and tetrahydrofuran (THF) were dried with $\mathrm{P}_{2} \mathrm{O}_{5}$, or $\mathrm{CaH}_{2}$, and purified by distillation before use. Reagent grade tetrabutylphosphonium bromide (TBPB), tetrabutylphosphonium chloride (TBPC), tetraphenylphosphonium chloride (TPPC), tetraphenylphosphonium bromide (TPPB), 18-crown-6 ether (18$\mathrm{C}-6)$, potassium chloride $(\mathrm{KCl})$, potassium bromide $(\mathrm{KBr})$, cesium fluoride $(\mathrm{CsF})$, cesium chloride $(\mathrm{CsCl})$, cesium bromide $(\mathrm{CsBr})$, potassium tert-butoxide $(t$ $\mathrm{BuOK})$, and potassium phenoxide $(\mathrm{PhOK})$ were dried

${ }^{\dagger}$ To whom correspondence should be addressed (E-mail: nishit02@kanagawa-u.ac.jp). 
in vacuo, and used without further purification. Commercial 4,4'-isopropylidenebis(2,6-dichlorophenol) (IBDP), acetyl chloride, benzoyl chloride, 2,4-dichlorobenzoyl chloride, and triethylamine (TEA) were used without further purification. Commercial 4,4'isopropylidenebisphenyl diacetate (BPAA) was recrystallized twice from methanol. Bis(oxetane)s, 1,4-bis[(3-ethyl-3-oxetanylmethoxymethyl)]benzene (BEOB), 1,4-bis[(3-ethy-3-oxetanyl)methoxy]benzene (1,4-BEOMB), 4,4'-bis[(3-ethyl-3-oxetanyl)methoxy]benzene (4,4'-BEOBP), 2,2' -bis[(3-ethyl-3oxetanyl)methoxy]biphenyl (2,2'-BEOBP), 3,3',5,5'tetramethyl-[4,4'-bis(3-ethyl-3-oxetanyl)methoxy]biphenyl (TM-BEOBP) were donated from Toagosei Co., Ltd. and were purified by recrystallization from appropriate solvents. 4,4'-Isopropylidenebisphenyl di-acetate (BPAA) was recrystallized twice from methanol.

\section{Measurement}

Melting points were determined with a Yanako MP500D hot-stage microscope. Infrared (IR) absorption spectra were measured on a Jasco model FT/IR-420 spectrometer. The NMR spectra were recorded on JNM FX-200 $\left(200 \mathrm{MHz}\right.$ for ${ }^{1} \mathrm{H}$ NMR and $50 \mathrm{MHz}$ for ${ }^{13} \mathrm{C}$ NMR) or JNM $\alpha-500$ (500 MHz for ${ }^{1} \mathrm{H}$ NMR and $125 \mathrm{MHz}$ for ${ }^{13} \mathrm{C} \mathrm{NMR}$ ) in $\mathrm{CDCl}_{3}$ with $\mathrm{Me}_{4} \mathrm{Si}$ (TMS) as an internal standard. The number-average molecular weight $\left(M_{\mathrm{n}}\right)$ and weight-average molecular weight $\left(M_{\mathrm{w}}\right)$ of the polymers were estimated by size exclusion chromatography (SEC; TOSOH model HLC-8020) on TSK gel Multipore Hxl-M columns calibrated by narrow molecular weight polystyrene standards and with tetrahydrofuran (THF) as an eluent without correction using a refractive index detector.

\section{Synthesis of Active Bis(ester)}

A typical procedure for the synthesis of active bis(ester) 4,4'-isopropylidenebis-(2,6-dichlorophenyl diacetate) (IBDPA) is as follows. Acetyl chloride $(12.8 \mathrm{~mL}, 180 \mathrm{mmol})$ in THF $(50 \mathrm{~mL})$ was added slowly to the solution of IBDP $(28.48 \mathrm{~g}, 80 \mathrm{mmol})$ and TEA $(30 \mathrm{~mL}, 216 \mathrm{mmol})$ in THF $(50 \mathrm{~mL})$ at 0 ${ }^{\circ} \mathrm{C}$. The reaction mixture was stirred at $25^{\circ} \mathrm{C}$ for $8 \mathrm{~h}$. The resulting mixture was poured into water to remove reaction solvent, TEA, and TEA salts. The precipitated solid product was filtered, washed with water and dried in vacuo. The obtained product was purified by the recrystallization from the mixed solvent of MEK/hexane (v/v $=5 / 2)$ to obtain a colorless solid IDBPA; yield 56\%; $\mathrm{mp}=136.8-137.2^{\circ} \mathrm{C} . \quad$ IR (KBr): $1772(v \mathrm{C}=\mathrm{O}$ of ester), $1468(v \mathrm{C}=\mathrm{C}$ of aromatic), $1187 \mathrm{~cm}^{-1}$ (v C-O-C). ${ }^{1} \mathrm{H}$ NMR $(200 \mathrm{MHz}$, $\left.\mathrm{CDCl}_{3}, \mathrm{TMS}\right) \delta=1.64\left(\mathrm{~s}, 6.0 \mathrm{H}, \mathrm{CH}_{3}\right), 2.39(\mathrm{~s}$, $\left.6.0 \mathrm{H}, \mathrm{CO}-\mathrm{CH}_{3}\right), 7.18 \mathrm{ppm}(\mathrm{s}, 4.0 \mathrm{H}$, aromatic $\mathrm{H})$.
${ }^{13} \mathrm{C}$ NMR $\left(50 \mathrm{MHz}, \mathrm{CDCl}_{3}, \mathrm{TMS}\right) \delta=20.22\left(\mathrm{CH}_{3}-\right.$ $\mathrm{CO}), 30.38\left(\mathrm{CH}_{3}\right), 42.85(-\mathrm{C}-), 127,00,128.67$, $142.30,148,56$ (aromatic C), $167.33 \mathrm{ppm}(\underline{\mathrm{C}}=\mathrm{O}) . \mathrm{El}$ em. Anal. Calcd for $\mathrm{C}_{19} \mathrm{H}_{16} \mathrm{O}_{4} \mathrm{Cl}_{4}: \mathrm{C}, 50.70 \% ; \mathrm{H}$, $3.58 \%$. Found: C, $50.60 \%$; $\mathrm{H}, 3.31 \%$.

\section{4,4'-Isopropylidenebis-(2,6-dichlorophenyl dibenzo- ate) (IBDPB)}

Yield $=45 \% . \quad \mathrm{Mp}=203.6-204.1^{\circ} \mathrm{C} . \quad$ IR $\quad(\mathrm{KBr})$ : 1750 ( $v \mathrm{C}=\mathrm{O}$ of ester), 1469 ( $v \mathrm{C}=\mathrm{C}$ of aromatic), $1242 \mathrm{~cm}^{-1}$ (v C-O-C), $809 \mathrm{~cm}^{-1}(v \mathrm{C}-\mathrm{Cl}) .{ }^{1} \mathrm{H}$ NMR $\left(200 \mathrm{MHz}, \mathrm{CDCl}_{3}, \mathrm{TMS}\right) \delta=1.57\left(\mathrm{~s}, 6.0 \mathrm{H}, \mathrm{CH}_{3}\right)$, $7.26(\mathrm{~s}, 4.0 \mathrm{H}$, aromatic $\mathrm{H}$ in bisphenol), 7.48-7.79 $(\mathrm{m}, 6.0 \mathrm{H}$, aromatic $\mathrm{H}$ in benzoate $), 8.29 \mathrm{ppm}(\mathrm{d}$, $J=6.8 \mathrm{~Hz}, 4.0 \mathrm{H}$, aromatic $\mathrm{H}$ in benzoate). ${ }^{13} \mathrm{C} \mathrm{NMR}$ $\left(50 \mathrm{MHz}, \mathrm{CDCl}_{3}, \mathrm{TMS}\right) \quad \delta=30.44\left(\mathrm{CH}_{3}\right), 42.97$ $(-\mathrm{C}-), \quad 127,06, \quad 128.19, \quad 128.70,128.95,129,63$, 130.53, 134.09, 148.71 (aromatic C), $163.18 \mathrm{ppm}$ $(\mathrm{C}=\mathrm{O})$. Elem. Anal. Calcd for $\mathrm{C}_{19} \mathrm{H}_{20} \mathrm{O}_{4} \mathrm{Cl}_{4}$ : C, $60.65 \%$; H, 3.51\%. Found: C, 60.48\%; H, 3.22\%.

\section{4,4'-Isopropylidenebis-(2,6-dichlorophenyl di-2,4-di- chlorobenzate) (IBDPD)}

Yield $=56 \% . \quad \mathrm{Mp}=189.4-189.9^{\circ} \mathrm{C} . \quad$ IR $\quad(\mathrm{KBr})$ : 1775 ( $v \mathrm{C}=\mathrm{O}$ of ester), 1473 ( $v \mathrm{C}=\mathrm{C}$ of aromatic), $1234 \mathrm{~cm}^{-1}$ (v C-O-C), $810 \mathrm{~cm}^{-1}$ (v C-Cl). ${ }^{1} \mathrm{H}$ NMR $\left(200 \mathrm{MHz}, \mathrm{CDCl}_{3}, \mathrm{TMS}\right) \delta=1.69\left(\mathrm{~s}, 6.0 \mathrm{H}, \mathrm{CH}_{3}\right)$, $7.26(\mathrm{~s}, 4.0 \mathrm{H}$, aromatic $\mathrm{H}$ in bisphenol), $7.42(\mathrm{dd}$, $J_{\mathrm{bc}}=2.0 \mathrm{~Hz}, J_{\mathrm{bd}}=8.3 \mathrm{~Hz}, 2.0 \mathrm{H}$, aromatic $\mathrm{H}$ in $2,4-$ dichlorobenzoate), $7.58\left(\mathrm{~d}, J_{\mathrm{cb}}=2.0 \mathrm{~Hz}, 2.0 \mathrm{H}\right.$, aromatic $\mathrm{H}$ in 2,4-dichlorobenzoate), $8.16 \mathrm{ppm}\left(\mathrm{d}, J_{\mathrm{db}}=\right.$ $8.3 \mathrm{~Hz}, 2.0 \mathrm{H}$, aromatic $\mathrm{H}$ in 2,4-dichlorobenzoate). ${ }^{13} \mathrm{C}$ NMR $\left(50 \mathrm{MHz}, \mathrm{CDCl}_{3}\right.$, TMS $) \delta=30.39\left(\mathrm{CH}_{3}\right)$, 43.03 (-C-), 126,06, 127.14, 127.29, 127.43, 128.84, $131.47,133.48,136.28,139.76,149.02$ (aromatic C), $160.76 \mathrm{ppm}(\underline{\mathrm{C}}=\mathrm{O})$. Elem. Anal. Calcd for $\mathrm{C}_{29} \mathrm{H}_{16^{-}}$ $\mathrm{O}_{4} \mathrm{Cl}_{8}$ : C, 48.92\%; H, 2.27\%. Found: C, 49.12\%; H, $2.03 \%$.

\section{Polyaddition of Bis(oxetane)s with Active Bis(ester)s}

A typical procedure for the polyaddition of BEOB with IDBPA is as follows: BEOB $(0.334 \mathrm{~g} ; 1.0 \mathrm{mmol})$, IBDPA $(0.450 \mathrm{~g} ; 1.0 \mathrm{mmol})$, TPPB $(0.021 \mathrm{~g} ; 0.05$ mmol), and DMAc $(0.3 \mathrm{~mL})$ were charged to a glass tube in a dry box, after which the tube was evacuated and sealed using a gas torch. The reaction was performed at $160{ }^{\circ} \mathrm{C}$ for $96 \mathrm{~h}$ in the sealed tube under stirring, and the tube opened. The resulting solution was diluted with a small amount of $\mathrm{CHCl}_{3}$ and poured into methanol $(100 \mathrm{~mL})$ to precipitate the polymer. The resulting polymer was precipitated twice from $\mathrm{CHCl}_{3}$ into excess methanol, and dried in vacuo at $60^{\circ} \mathrm{C}$ for $24 \mathrm{~h}$. The yield of poly(BEOB-IBDPA) was $0.737 \mathrm{~g} \mathrm{(94 \% ).} \mathrm{The} \mathrm{number-average} \mathrm{moleculer} \mathrm{weight}$ $\left(M_{\mathrm{n}}\right)$ of the polymer estimated by SEC was 7200 
$\left(M_{\mathrm{w}} / M_{\mathrm{n}}=1.67\right) . \mathrm{IR}($ film): $1740(v \mathrm{C}=\mathrm{O}$, ester $)$, 1472 ( $v \mathrm{C}=\mathrm{C}$ of aromatic), 1274 ( $v \mathrm{C}=\mathrm{O}$, ester), 1098 ( $v$ C-O-C, ether), $801 \mathrm{~cm}^{-1}(v \mathrm{C}-\mathrm{Cl}) .{ }^{1} \mathrm{H}$ NMR $\left(500 \mathrm{MHz}, \mathrm{CDCl}_{3}, \mathrm{TMS}\right) \delta=0.95(\mathrm{t}, J=7.5 \mathrm{~Hz}$, $6.0 \mathrm{H}, \mathrm{CH}_{2} \mathrm{CH}_{3}$ ), 1.57 (s, 6.0H, C- $\mathrm{CH}_{3}$ ), 1.63 (q, $J=$ $\left.7.5 \mathrm{~Hz}, 4.0 \mathrm{H}, \mathrm{CH}_{2} \mathrm{CH}_{3}\right) 2.02\left(\mathrm{~s}, 6.0 \mathrm{H}, \mathrm{COCH}_{3}\right), 3.52-$ $3.56\left(\mathrm{~m}, 4.0 \mathrm{H}, \mathrm{O}-\mathrm{CH}_{2}-\mathrm{C}\right), 3.96$ (s, $4.0 \mathrm{H}, \mathrm{O}-\mathrm{CH}_{2}-\mathrm{Ar}-$ $\mathrm{CH}_{2}-\mathrm{O}$ ), 4.20-4.23 (m, 4.0H, C- $\mathrm{CH}_{2}-\mathrm{O}-\mathrm{Ar}-\mathrm{O}-\mathrm{CH}_{2}-$ C), 4.50 (s, $\left.4.0 \mathrm{H}, \mathrm{C}-\mathrm{CH}_{2}-\mathrm{O}-\mathrm{CO}\right), 7.06$ (s, $4.0 \mathrm{H}$, aromatic $\mathrm{H}$ in bisphenol), $7.28 \mathrm{ppm}$ (s, $4.0 \mathrm{H}, \mathrm{CH}_{2}-\mathrm{C}_{6} \underline{\mathrm{H}}_{4}-$ $\left.\mathrm{CH}_{2}\right) . \quad{ }^{13} \mathrm{C} \mathrm{NMR} \quad\left(125 \mathrm{MHz}, \quad \mathrm{CDCl}_{3}\right) . \quad \delta=7.6$ $\left(\mathrm{CH}_{2} \underline{\mathrm{CH}}_{3}\right), 20.9 \quad\left(\mathrm{C}-\underline{\mathrm{CH}_{3}}\right), 22.7 \quad\left(\mathrm{CH}_{2}-\mathrm{CH}_{3}\right), 30.3$ $\mathrm{COCH}_{3}$ ), 42.4 (quaternary carbon in bisphenol unit), 42.7 (quaternary carbon in methylene unit), 64.6 (C$\mathrm{CH}_{2}-\mathrm{O}-$ bisphenol $), 69.8\left(\mathrm{C}-\mathrm{CH}_{2}-\mathrm{O}-\mathrm{CH}_{2}-\mathrm{Ar}-\mathrm{CH}_{2}-\right.$ $\left.\mathrm{O}-\mathrm{CH}_{2}-\mathrm{C}\right), 72.8\left(\mathrm{C}-\mathrm{CH}_{2}-\mathrm{O}-\mathrm{CO}\right), 73.2\left(\mathrm{O}-\mathrm{CH}_{2}-\mathrm{Ar}-\right.$ $\mathrm{CH}_{2}-\mathrm{O}$ ), 127.3, 127.4, 129.1, 137.8, 146.7, 149.1 (aromatic C), $170.9 \mathrm{ppm}\left(\mathrm{O}-\mathrm{CO}-\mathrm{CH}_{3}\right)$. Elem. Anal. Calcd for $\mathrm{C}_{33} \mathrm{H}_{49} \mathrm{O}_{8} \mathrm{Cl}_{4}$ : C, 59.69\%; H, 5.92\%. Found: C, $59.67 \% ; \mathrm{H}, 5.83 \%$.

\section{Poly (1,4-BEOMB-IBDPA)}

Yield $=0.745 \quad(99 \%) . \quad M_{\mathrm{n}}=11200, \quad M_{\mathrm{w}} / M_{\mathrm{n}}=$ 1.88. IR (film): 1743 ( $v \mathrm{C}=\mathrm{O}$, ester), $1508(v \mathrm{C}=\mathrm{C}$, aromatic), 1127 ( $v \mathrm{C}-\mathrm{O}-\mathrm{C}$ ester), 1034 ( $v \mathrm{C}-\mathrm{O}-\mathrm{C}$, ether $), 801 \mathrm{~cm}^{-1} \quad(v \mathrm{C}-\mathrm{Cl}) .{ }^{1} \mathrm{H}$ NMR $(500 \mathrm{MHz}$, $\left.\mathrm{CDCl}_{3}, \mathrm{TMS}\right) \delta=1.01\left(\mathrm{t}, J=7.0 \mathrm{~Hz}, 6.0 \mathrm{H}, \mathrm{CH}_{2-}\right.$ $\left.\mathrm{C}_{3}\right), 1.57$ (s, $\left.6.0 \mathrm{H}, \mathrm{C}-\mathrm{C}_{3}\right), 1.77(\mathrm{q}, J=7.0 \mathrm{~Hz}$, $\left.4.0 \mathrm{H}, \mathrm{C}_{2} \mathrm{CH}_{3}\right) 2.06$ (s, $\left.6.0 \mathrm{H}, \mathrm{COC}_{3}\right), 4.00-4.05$ (m, $8.0 \mathrm{H}, \quad \mathrm{Ar}-\mathrm{O}-\mathrm{C}_{2}-\mathrm{C}-\mathrm{C}_{2}-\mathrm{O}-$ bisphenol unit), $4.30\left(\mathrm{~s}, 4.0 \mathrm{H}, \mathrm{C}-\mathrm{CH}_{2}-\mathrm{O}-\mathrm{CO}\right), 6.84(\mathrm{~s}, 4.0 \mathrm{H}, \mathrm{O}-$ $\left.\mathrm{C}_{6} \underline{\mathrm{H}}_{4}-\mathrm{O}\right), 7.06 \mathrm{ppm}(\mathrm{s}, 4.0 \mathrm{H}$, aromatic $\mathrm{H}$ in bisphenol). $\quad{ }^{13} \mathrm{C}$ NMR $\quad\left(125 \mathrm{MHz}, \quad \mathrm{CDCl}_{3}\right) . \quad \delta=7.6$ $\left(\mathrm{CH}_{2} \underline{\mathrm{CH}}_{3}\right), 20.9\left(\mathrm{C}-\underline{\mathrm{CH}}_{3}\right), 22.7\left(\underline{\mathrm{CH}}_{2}-\mathrm{CH}_{3}\right), 30.3$ $\mathrm{CO} \mathrm{CH}_{3}$ ), 42.4 (quaternary carbon in bisphenol unit), 42.5 (quaternary carbon in methylene unit), 64.3 (C$\mathrm{CH}_{2}-\mathrm{O}$-bisphenol), 68.0 (C- $\left.\mathrm{CH}_{2}-\mathrm{O}-\mathrm{Ar}-\mathrm{O}-\mathrm{CH}_{2}-\mathrm{C}\right)$, $72.5\left(\mathrm{C}-\mathrm{CH}_{2}-\mathrm{O}-\mathrm{CO}\right), 115.3,127.3,129.1,146.8$, 149.0, 153.3 (aromatic C), 170.9 ppm (O- $\mathrm{CO}-\mathrm{CH}_{3}$ ). Elem. Anal. Calcd for $\mathrm{C}_{37} \mathrm{H}_{42} \mathrm{O}_{8} \mathrm{Cl}_{4}$ : C, $58.73 \% ; \mathrm{H}$, $5.61 \%$. Found: C, $58.52 \%$; H, $5.56 \%$.

\section{Poly $\left(4,4^{\prime}-B E O B P-I B D P A\right)$}

Yield $=0.791 \quad(95 \%) . \quad M_{\mathrm{n}}=17400, \quad M_{\mathrm{w}} / M_{\mathrm{n}}=$ 1.78. IR (film): 1742 ( $v \mathrm{C}=\mathrm{O}$, ester), $1499(v \mathrm{C}=\mathrm{C}$, aromatic), 1237 ( $v \mathrm{C}-\mathrm{O}-\mathrm{C}$ ester), 1038 ( $v \mathrm{C}-\mathrm{O}-\mathrm{C}$, ether), $801 \mathrm{~cm}^{-1} \quad(v \mathrm{C}-\mathrm{Cl}) .{ }^{1} \mathrm{H} \mathrm{NMR} \quad(500 \mathrm{MHz}$, $\left.\mathrm{CDCl}_{3}, \mathrm{TMS}\right) \delta=1.03\left(\mathrm{t}, J=7.3 \mathrm{~Hz}, 6.0 \mathrm{H}, \mathrm{CH}_{2}-\right.$ $\left.\mathrm{C}_{3}\right), 1.57\left(\mathrm{~s}, 6.0 \mathrm{H}, \mathrm{C}-\mathrm{C}_{3}\right), 1.78(\mathrm{q}, J=7.3 \mathrm{~Hz}$, $\left.4.0 \mathrm{H}, \mathrm{CH}_{2} \mathrm{CH}_{3}\right) 2.06\left(\mathrm{~s}, 6.0 \mathrm{H}, \mathrm{COCH}_{3}\right), 4.08$ (s, $4.0 \mathrm{H}, \mathrm{C}-\mathrm{C}_{2}-\mathrm{O}-$ biphenyl unit), 4.10 (s, $4.0 \mathrm{H}, \mathrm{C}-$ $\mathrm{CH}_{2}-\mathrm{O}-$ bisphenol unit), 4.28-4.33 (m, 4.0H, C$\left.\mathrm{C}_{2}-\mathrm{O}-\mathrm{CO}\right), 6.96$ (d, $\left.J=8.7 \mathrm{~Hz}, 4.0 \mathrm{H}, \mathrm{C}_{12} \underline{\mathrm{H}}_{4}-\mathrm{O}\right)$, $7.06(\mathrm{~s}, 4.0 \mathrm{H}$, aromatic $\mathrm{H}$ in bisphenol), $7.46 \mathrm{ppm}$ $\left(\mathrm{d}, J=8.7 \mathrm{~Hz}, \quad 4.0 \mathrm{H}, \quad \mathrm{C}_{12} \underline{\mathrm{H}}_{4}-\mathrm{O}\right) .{ }^{13} \mathrm{C}$ NMR $(125$
$\left.\mathrm{MHz}, \mathrm{CDCl}_{3}\right) . \delta=7.7\left(\mathrm{CH}_{2} \underline{\mathrm{CH}}_{3}\right), 20.9\left(\mathrm{C}-\underline{\mathrm{CH}}_{3}\right)$, $\left.22.7\left(\mathrm{CH}_{2}-\mathrm{CH}_{3}\right), 30.3 \mathrm{COCH}_{3}\right), 42.4$ (quaternary carbon in bisphenol unit), 42.5 (quaternary carbon in methylene unit), 64.3 (C- $\underline{\mathrm{CH}}_{2}-\mathrm{O}-$ bisphenol), 64.5 (C- $\mathrm{CH}_{2}-\mathrm{O}-$ biphenyl-O- $\left.\underline{\mathrm{CH}}_{2}-\mathrm{C}\right), 72.4 \quad\left(\mathrm{C}-\mathrm{CH}_{2}-\mathrm{O}-\right.$ CO), 114.8, 127.3, 127.7, 129.2, 133.6, 146.8, 149.0, 158.1 (aromatic C), 170.9 ppm (O-CO- $\left.\mathrm{CH}_{3}\right)$. Elem. Anal. Calcd for $\mathrm{C}_{42} \mathrm{H}_{46} \mathrm{O}_{8} \mathrm{Cl}_{4}: \mathrm{C}, 62.05 \%$;, $5.57 \%$. Found: C, $62.04 \%$; H, 5.47\%.

\section{Poly $\left(2,2^{\prime}-B E O B P-I B D P A\right)$}

Yield $=0.750 \quad(90 \%) . \quad M_{\mathrm{n}}=11400, \quad M_{\mathrm{w}} / M_{\mathrm{n}}=$ 1.69. IR (film): 1742 ( $v \mathrm{C}=\mathrm{O}$, ester), $1498(v \mathrm{C}=\mathrm{C}$, aromatic), 1236 ( $v \mathrm{C}-\mathrm{O}-\mathrm{C}$ ester), 1034 ( $v \mathrm{C}-\mathrm{O}-\mathrm{C}$, ether), $801 \mathrm{~cm}^{-1} \quad(v \mathrm{C}-\mathrm{Cl}) .{ }^{1} \mathrm{H} \mathrm{NMR} \quad(500 \mathrm{MHz}$, $\left.\mathrm{CDCl}_{3}, \mathrm{TMS}\right) \delta=0.78\left(\mathrm{t}, J=6.9 \mathrm{~Hz}, 6.0 \mathrm{H}, \mathrm{CH}_{2}-\right.$ $\left.\mathrm{C}_{3}\right), 1.45-1.57\left(\mathrm{~m}, 10.0 \mathrm{H}, \mathrm{C}-\mathrm{C}_{3}\right.$ and $\left.\mathrm{CH}_{2} \mathrm{CH}_{3}\right)$, 1.98 (s, $\left.6.0 \mathrm{H}, \mathrm{COCH}_{3}\right), 3.77$ (s, $4.0 \mathrm{H}, \mathrm{C}-\mathrm{C}_{2}-\mathrm{O}-$ biphenyl unit), $3.95\left(\mathrm{~s}, 4.0 \mathrm{H}, \mathrm{C}-\mathrm{C}_{2}-\mathrm{O}-\right.$ bisphenol unit), 4.06 (s, 4.0H, C- $\underline{\mathrm{H}}_{2}-\mathrm{O}-\mathrm{CO}$ ), 6.87-6.94 (m, $\left.4.0 \mathrm{H}, \mathrm{C}_{12} \underline{\mathrm{H}}_{4}-\mathrm{O}\right), 7.04$ (s, $4.0 \mathrm{H}$, aromatic $\mathrm{H}$ in bisphenol), 7.12-7.21 ppm (d, 4.0H, $\left.\mathrm{C}_{12} \underline{\mathrm{H}}_{4}-\mathrm{O}\right) .{ }^{13} \mathrm{C}$ NMR $\left(125 \mathrm{MHz}, \mathrm{CDCl}_{3}\right) . \quad \delta=7.5 \quad\left(\mathrm{CH}_{2} \mathrm{CH}_{3}\right), 20.8(\mathrm{C}-$ $\left.\left.\mathrm{CH}_{3}\right), 22.7\left(\mathrm{CH}_{2}-\mathrm{CH}_{3}\right), 30.3 \mathrm{COCH}_{3}\right), 42.4$ (quaternary carbon in bisphenol unit), 42.5 (quaternary carbon in methylene unit), $64.6\left(\mathrm{C}-\mathrm{CH}_{2}-\mathrm{O}-\right.$ bisphenol $)$, $68.1 \quad\left(\mathrm{C}-\mathrm{CH}_{2}-\mathrm{O}-\right.$ biphenyl-O- $\left.{ }^{-} \mathrm{CH}_{2}-\mathrm{C}\right), \quad 72.7 \quad(\mathrm{C}-$ $\left.\mathrm{CH}_{2}-\mathrm{O}-\mathrm{CO}\right), 112.1,120.3,127.2,128.1,128.4$, $129.1,131.2,146.7,149.0,156.1$ (aromatic C), $170.7 \mathrm{ppm}\left(\mathrm{O}-\mathrm{CO}-\mathrm{CH}_{3}\right)$. Elem. Anal. Calcd for $\mathrm{C}_{42} \mathrm{H}_{46} \mathrm{O}_{8} \mathrm{Cl}_{4}: \mathrm{C}, 62.05 \%$; $\mathrm{H}, 5.57 \%$. Found: $\mathrm{C}$, $61.87 \%$; H, $5.49 \%$.

\section{Poly (TM-BEOBP-IBDPA)}

Yield $=0.809 \quad(91 \%) . \quad M_{\mathrm{n}}=12500, \quad M_{\mathrm{w}} / M_{\mathrm{n}}=$ 1.88. IR (film): 1739 ( $v \mathrm{C}=\mathrm{O}$, ester), $1471(v \mathrm{C}=\mathrm{C}$, aromatic), 1234 ( $v \mathrm{C}-\mathrm{O}-\mathrm{C}$ ester), 1022 ( $v \mathrm{C}-\mathrm{O}-\mathrm{C}$, ether), $800 \mathrm{~cm}^{-1}$ (v $\left.\mathrm{C}-\mathrm{Cl}\right) .{ }^{1} \mathrm{H}$ NMR $(500 \mathrm{MHz}$, $\left.\mathrm{CDCl}_{3}, \mathrm{TMS}\right) \delta=1.11\left(\mathrm{t}, J=6.9 \mathrm{~Hz}, 6.0 \mathrm{H}, \mathrm{CH}_{2}^{-}\right.$ $\left.\mathrm{C}_{3}\right), 1.60\left(\mathrm{~s}, 6.0 \mathrm{H}, \mathrm{C}-\mathrm{CH}_{3}\right), 1.88(\mathrm{q}, J=6.9 \mathrm{~Hz}$, $\left.4.0 \mathrm{H}, \mathrm{CH}_{2} \mathrm{CH}_{3}\right), 2.09$ (s, $\left.6.0 \mathrm{H}, \mathrm{COCH}_{3}\right), 2.32(\mathrm{~s}$, $\left.12.0 \mathrm{H}, \mathrm{Ar}-\mathrm{CH}_{3}\right), 3.89$ (s, 4.0H, C- $\mathrm{CH}_{2}-\mathrm{O}-$ biphenyl unit), 4.14 (s, $4.0 \mathrm{H}, \mathrm{C}-\mathrm{CH}_{2}-\mathrm{O}-$ bisphenol unit), 4.46 (s, $\left.4.0 \mathrm{H}, \mathrm{C}-\mathrm{CH}_{2}-\mathrm{O}-\mathrm{CO}\right), 7.09$ (s, $4.0 \mathrm{H}$, aromatic $\mathrm{H}$ in bisphenol), $7.14 \mathrm{ppm}(\mathrm{s}, 4.0 \mathrm{H}$, aromatic $\mathrm{H}$ in biphenyl). ${ }^{13} \mathrm{C}$ NMR $\left(125 \mathrm{MHz}, \mathrm{CDCl}_{3}\right) . \quad \delta=7.9$ $\left(\mathrm{CH}_{2} \mathrm{CH}_{3}\right), \quad 16.5 \quad\left(\mathrm{Ar}-\mathrm{CH}_{3}\right), 21.0 \quad\left(\mathrm{C}-\mathrm{CH}_{3}\right), 23.0$ $\left.\left(\mathrm{CH}_{2}-\mathrm{CH}_{3}\right), 30.3 \mathrm{CO}^{-} \mathrm{H}_{3}\right), 42.4$ (quaternary carbon in bisphenol unit), 43.0 (quaternary carbon in methylene unit), 65.0 (C- $\mathrm{CH}_{2}-\mathrm{O}-$ bisphenol-O- $\left.\underline{\mathrm{CH}}_{2}-\mathrm{C}\right)$, $71.4\left(\mathrm{C}-\mathrm{CH}_{2}-\mathrm{O}-\right.$ biphenyl $), 72.9 \quad\left(\mathrm{C}-\mathrm{CH}_{2}-\mathrm{O}-\mathrm{CO}\right)$, 127.4, 127.6, 131.0, 136.5, 146.7, 149.1, 154.5, (aromatic C), 170.9 ppm (O-CO- $\left.\mathrm{CH}_{3}\right)$. Elem. Anal. Calcd for $\mathrm{C}_{46} \mathrm{H}_{54} \mathrm{O}_{8} \mathrm{Cl}_{4}$ : C, $63.36 \%$; H, 6.52\%. Found: C, $63.07 \%$; H, 6.34\%. 

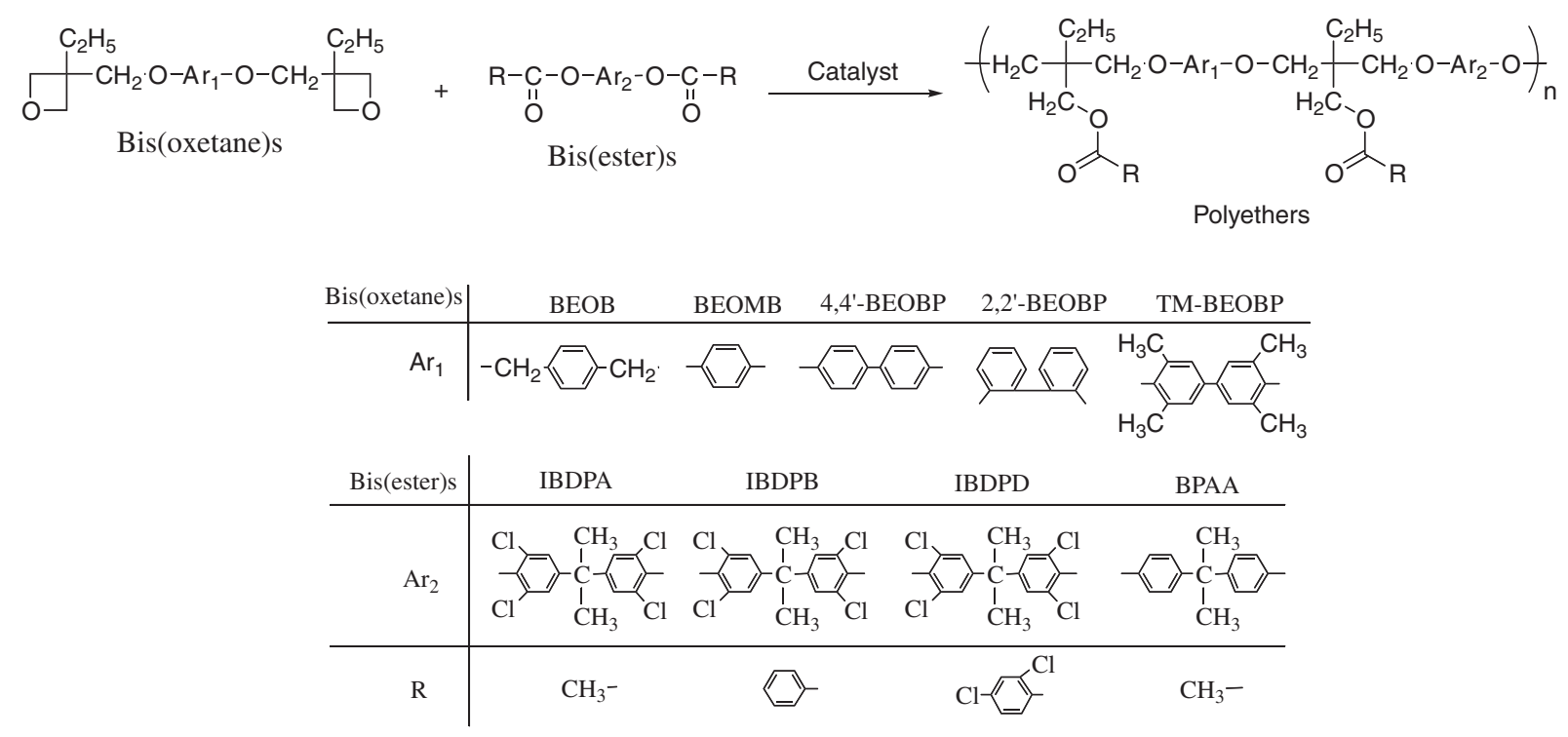

Scheme 1.

\section{RESULTS AND DISCUSSION}

It has been found that addition reactions of oxetanes with carboxylic acids, thiols, phenols, and $S$-phenyl thioesters proceed selectively and smoothly without any side-reaction under appropriate reaction conditions using certain quaternary onium salts or crown ether complexes as catalysts. Therefore, we examine to develop new addition reaction of oxetanes with phenyl esters in this article.

Active esters IBDPA, IBDPB, and IBDPD were obtained in 56,45 , and $56 \%$ yields, respectively, by the reaction of IBDP with acetyl chloride, benzoyl chloride, and 2,4-dichlorobenzoyl chloride. Then, the resulting products were confirmed by IR and NMR spectra, and elemental analysis. Commercial BPAA was also examined as an active bis(ester).

Since the polyaddition ${ }^{14}$ of bis(oxetane)s with di-( $s$ phenyl thioester)s proceeded at higher temperature than $150^{\circ} \mathrm{C}$ to produce corresponding polyesters with high molecular weight, the polyaddition of bis(oxetane)s with the above active bis(ester)s were performed at $160^{\circ} \mathrm{C}$ using the same catalyst system. The reaction of bis(oxetane) 4,4'-BEOBP with IBDPA did not proceed without catalyst in DMAc at $160^{\circ} \mathrm{C}$ for $96 \mathrm{~h}$. However, the polyaddition proceeded smoothly, and corresponding poly(ether)s with $M_{\mathrm{n}}$ 's $=9200-17400$ were obtained in $92-95 \%$ yields when $5 \mathrm{~mol} \%$ of TBPC, TBPB, TPPC or TPPB were used as catalysts (Table I). In this reaction system, tetraphenylphosphonium salts TBPB and TBPC showed higher catalytic activity than tetrabutylphosphonium salts TBPC and TBPB, because the former catalysts have good thermal stabilities. ${ }^{26-29}$
The structure of the obtained polymer was confirmed by the IR, ${ }^{1} \mathrm{H}$ NMR and ${ }^{13} \mathrm{C}$ NMR spectroscopy, and the elemental analysis. In the IR spectrum of the polymer film, the absorption peaks at 1772 and $1187 \mathrm{~cm}^{-1}$ assignable to the $\mathrm{C}=\mathrm{O}$ and $\mathrm{C}-\mathrm{O}-\mathrm{C}$ stretching from aryl ester linkage disappeared, and shows new appearance of characteristic absorption peaks at 1742 and $1237 \mathrm{~cm}^{-1}$ assignable to the $\mathrm{C}=\mathrm{O}$ and $\mathrm{C}-$ O-C (ester) from alkyl ester linkage, respectively. The ${ }^{1} \mathrm{H}$ NMR spectrum of this polymer shows signals at $\delta=1.03,1.57$ due to both $-\mathrm{CH}_{3}, 1.78$ due to $-\mathrm{C}_{2} \mathrm{CH}_{3}, 2.06$ due to $\mathrm{COCH}_{3}, 4.08$ due to $\mathrm{C}-\underline{\mathrm{C}}_{2}-$ O-biphenyl unit, 4.10 due to $\mathrm{C}-\mathrm{C}_{2}-\mathrm{O}$-bisphenol unit, 4.28-4.33 due to $\mathrm{C}-\mathrm{CH}_{2}-\mathrm{O}-\mathrm{CO}$, and 6.96, 7.06 and $7.46 \mathrm{ppm}$ due to aromatic protons. These results show that the polyaddition of bis(oxetane) 4,4'BEOBP with active bis(ester) IBDPA proceeded to afford the poly(ether) poly(4,4'-BEOBP-IDBPA), whose structure is shown in Scheme 1 . The ${ }^{13} \mathrm{C}$ NMR spectrum also supported this structure.

We also examined the catalytic activity of crown ether complexes such as $18-\mathrm{C}-6 / \mathrm{KCl}, 18-\mathrm{C}-6 / \mathrm{KBr}$, 18-C-6/CsF, $18-\mathrm{C}-6 / \mathrm{CsCl}, 18-\mathrm{C}-6 / \mathrm{CsBr}, 18-\mathrm{C}-6 / t$ $\mathrm{BuOK}$, and $18-\mathrm{C}-6 / \mathrm{PhOK}$ under the same conditions. As summarized in Table I, the polyaddition of 4,4'BEOBP with IBDPA proceeded when these crown complexes were used as catalysts. However, molecular weights and yields of the resulting poly(ether)s were not high enough. In addition, SEC of these obtained polymers showed multimodal curves. Although the complexes $18-\mathrm{C}-6 / \mathrm{CsF}, 18-\mathrm{C}-6 / t-\mathrm{BuOK}$ and 18 $\mathrm{C}-6 / \mathrm{PhOK}$ showed relatively higher activity than other crown ether complexes, the activities of these crown ether complexes were lower than those of the above quaternary phosphonium salts under the same 
Polyaddition of Bis(oxetane)s with Active Bis(ester)s

Table I. The polyaddition of $4,4^{\prime}$-BEOBP with IBDPA using certain catalysts ${ }^{\mathrm{a}}$

\begin{tabular}{clccc}
\hline Run no. & \multicolumn{1}{c}{ Catalyst } & $\begin{array}{c}\text { Yield } \\
(\%)^{\mathrm{b}}\end{array}$ & $M_{\mathrm{n}}{ }^{\mathrm{c}}$ & $M_{\mathrm{W}} / M_{\mathrm{n}}$ \\
\hline 1 & No catalyst & 0 & - & - \\
2 & TBPC & 92 & 9200 & 1.45 \\
3 & TBPB & 92 & 10800 & 1.82 \\
4 & TPPC & 93 & 13400 & 1.59 \\
5 & TPPB & 95 & 17400 & 1.78 \\
6 & $18-\mathrm{C}-6 / \mathrm{KCl}$ & 49 & $3800,2400,1800$ & multimodal \\
7 & $18-\mathrm{C}-6 / \mathrm{KBr}$ & 39 & $2700,1800,1300$ & multimodal \\
8 & $18-\mathrm{C}-6 / \mathrm{CsF}$ & 80 & $5000,2600,1800$ & multimodal \\
9 & $18-\mathrm{C}-6 / \mathrm{CsCl}$ & 27 & $2900,1900,1300$ & multimodal \\
10 & $18-\mathrm{C}-6 / \mathrm{CsBr}$ & 24 & $2600,1900,1300$ & multimodal \\
11 & $18-\mathrm{C}-6 / t-\mathrm{BuOK}$ & 73 & $4000,2300,1800$ & multimodal \\
12 & $18-\mathrm{C}-6 / \mathrm{PhOK}$ & 82 & $4800,2900,2300$ & multimodal \\
\hline
\end{tabular}

${ }^{\text {a }}$ The reaction was carried out with $4,4^{\prime}$-BEOBP $(1.0 \mathrm{mmol})$ and IBDPA $(1.0 \mathrm{mmol})$ using certain catalyst $(5 \mathrm{~mol} \%)$ in DMAc $(2 \mathrm{~mol} / \mathrm{L})$ at $160{ }^{\circ} \mathrm{C}$ for $96 \mathrm{~h} .{ }^{\mathrm{b}}$ Insoluble part in methanol. ${ }^{\mathrm{c}}$ Estimated by SEC based on polystyrene standards.

Table II. The polyaddition of bis(oxetane) with certain active bis(ester)s in DMAc or NMPa

\begin{tabular}{|c|c|c|c|c|c|c|c|c|}
\hline \multirow[b]{2}{*}{ Run no. } & \multicolumn{6}{|c|}{ Condition } & \multirow[b]{2}{*}{$M_{\mathrm{n}}{ }^{\mathrm{c}}$} & \multirow[b]{2}{*}{$M_{\mathrm{w}} / M_{\mathrm{n}}$} \\
\hline & Bis(oxetane) & Bis(ester) & Solvent & $\begin{array}{l}\text { Temp. } \\
\left({ }^{\circ} \mathrm{C}\right)\end{array}$ & $\begin{array}{l}\text { Time } \\
\text { (h) }\end{array}$ & $\begin{array}{l}\text { Yield } \\
(\%)^{\mathrm{b}}\end{array}$ & & \\
\hline 1 & BEOB & BPAA & DMAc & 160 & 48 & 0 & - & - \\
\hline 2 & BEOB & IBDPA & DMAc & 160 & 48 & 90 & 7100 & 1.62 \\
\hline 3 & BEOB & IBDPB & DMAc & 160 & 48 & 0 & - & - \\
\hline 4 & BEOB & IBDPD & DMAc & 160 & 48 & 49 & 2500 & 1.04 \\
\hline 5 & 4,4'-BEOBP & IBDPA & DMAc & 160 & 96 & 95 & 17400 & 1.78 \\
\hline 6 & 4,4'-BEOBP & IBDPB & DMAc & 160 & 96 & 0 & - & - \\
\hline 7 & 4,4'-BEOBP & IBDPD & DMAc & 160 & 96 & 59 & $2700,1800,1100$ & multi. ${ }^{\mathrm{d}}$ \\
\hline 8 & 4,4'-BEOBP & IBDPB & NMP & 180 & 96 & 38 & $2600,1600,1000$ & multi. ${ }^{\mathrm{d}}$ \\
\hline 9 & 4,4'-BEOBP & IBDPD & NMP & 180 & 96 & 84 & 4500 & 1.85 \\
\hline
\end{tabular}

${ }^{\mathrm{a}}$ The reaction was carried out with bis(oxetane)s $(1.0 \mathrm{mmol})$ and bis(ester)s $(1.0 \mathrm{mmol})$ using TPPB $(5 \mathrm{~mol} \%)$ as a catalyst in the solvent $(2 \mathrm{~mol} / \mathrm{L}) .{ }^{\mathrm{b}}$ Insoluble materials in methanol. ${ }^{\mathrm{c}}$ Estimated by SEC based on polystyrene standards. ${ }^{\mathrm{d}}$ Multimodal.

conditions. This may be due to less solubility of the crown ether complexes into the reaction solvent.

Then, polyadditions of bis(oxetane) BEOB with such active bis(ester)s as IBDPA, IBDPD, IBDPB and BPAA were examined using $5 \mathrm{~mol} \%$ of TPPB as the catalyst in DMAc at $160^{\circ} \mathrm{C}$ for $48 \mathrm{~h}$. As summarized in Table II, the reaction of BEOB with BPAA and IBDPB did not proceed under this reaction condition (runs 1 and 3 in Table II). On the other hand, the reaction of BEOB with IBDPD proceeded to give low molecular weight oligomer with $M_{\mathrm{n}}=2500$ in $49 \%$ yield (run 4 in Table I). The reaction of BEOB with IBDPA proceeded smoothly, and the polymer with $M_{\mathrm{n}}=7100$ was obtained in $90 \%$ yield (run 2 in Table I). These mean that the reactivity of active bis(ester) on the reaction with bis(oxetane) was strongly affected by the electrophilicity of the esters. That is, IBDPA and IBDPD composed from high nu- cleophilic phenol unit such as IBDP and high nucleophilic carboxyl units such as acetyl and 2,4-dichlorobenzoyl ester groups have good reactivities (Scheme 1).

Polyadditions of 4,4'-BEOBP, which seems to be suitable structure to produce the polymer, with IBDPB and IBDPD were also examined under the same conditions applied for the polyaddition of 4,4'-BEOBP with IBDPA, using TPPB as the catalyst in DMAc at $160^{\circ} \mathrm{C}$ for $96 \mathrm{~h}$. Although the polyaddition of $4,4^{\prime}$-BEOBP with IBDPD gave the corresponding polymer in 59\% yield, the molecular weight of the resulting polymer is not high enough. On the other hand, the polyaddition of 4,4'-BEOBP with IBDPB did not proceed at all.

Therefore, the polyadditions of 4,4'-BEOBP with IBDPB and IBDPD were taken place in NMP at higher temperature such as $180^{\circ} \mathrm{C}$. Upon this reaction 


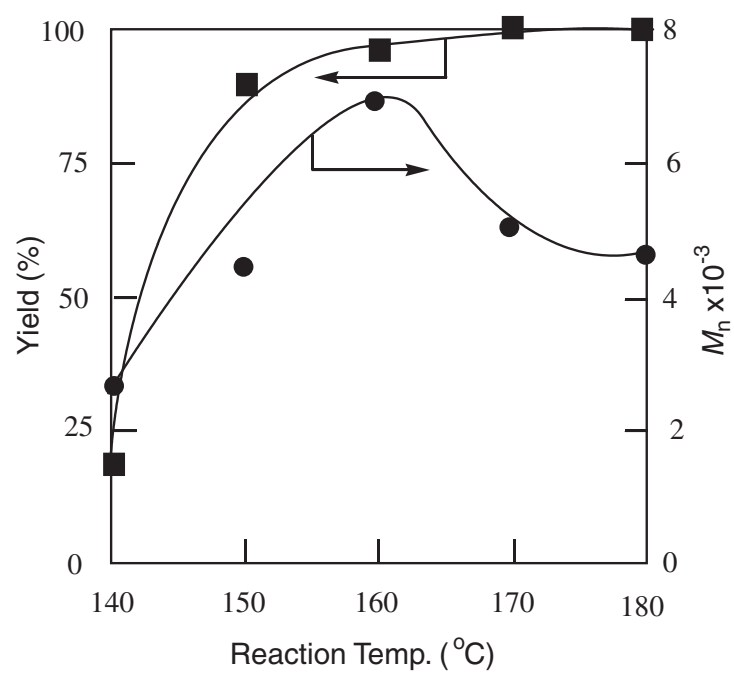

Figure 1. Effect of reaction temperature on the polyaddition of BEOB with IBDPA. The reaction was carried out with BEOB $(1.0 \mathrm{mmol})$ with IBDPA $(1.0 \mathrm{mmol})$ using TPPB $(5 \mathrm{~mol} \%)$ as the catalyst in DMAc $(2 \mathrm{~mol} / \mathrm{L})$ for $48 \mathrm{~h} .(\bullet)$ : Mn, (匹): Yield.

temperature, the polyaddition of 4,4'-BEOBP with IBDPB proceeded partly, and low molecular weight oligomer was obtained in $38 \%$ yield. The polyaddition of $4,4^{\prime}$-BEOBP with IBDPD was enhanced at $180^{\circ} \mathrm{C}$, and the corresponding polymer with $M_{\mathrm{n}}=4500$ was obtained in $84 \%$ yield (Table II).

The above results suggest that the yields and molecular weights of the polymers on the polyaddition of bis(oxetane)s with active bis(ester)s were also affected by the reaction time and temperature. Therefore, the effect of reaction temperature was examined on the polyaddition of BEOB with IBDPA using $5 \mathrm{~mol} \%$ of TPPB as a catalyst in DMAc for $48 \mathrm{~h}$ at several temperatures in the range between $140-180^{\circ} \mathrm{C}$. Figure 1 depicts the relationships of the yields, $M_{\mathrm{n}} \mathrm{s}$, and reaction temperatures. As shown in Figure 1, the yields of the polymers increased strongly with reaction temperature. The $M_{\mathrm{n}} \mathrm{s}$ of the polymers also increased until $160{ }^{\circ} \mathrm{C}$. However, at higher temperature than $160^{\circ} \mathrm{C}$, the $M_{\mathrm{n}} \mathrm{s}$ of the resulting polymers decreased gradually. It may be suggested that some decomposition of ether linkages of the polymer chain occurred. This result seems that $160{ }^{\circ} \mathrm{C}$ is a suitable reaction temperature on the polyaddition of bis(oxetane) with active bis(ester).

The effect of reaction time on the polyadditions of BEOB or 4,4'-BEOBP with IBDPA was examined using $5 \mathrm{~mol} \%$ of TPPB as the catalyst in DMAc at $160^{\circ} \mathrm{C}$ for various time. As shown in Figure 2, the yield of the polymer on the polyaddition of BEOB with IBDPA was relatively good for $24 \mathrm{~h}$, and we obtained the corresponding polymers quantitatively for more than $72 \mathrm{~h}$. On the other hand, the $M_{\mathrm{n}} \mathrm{s}$ of the polymers increased until $96 \mathrm{~h}$ of reaction. However,

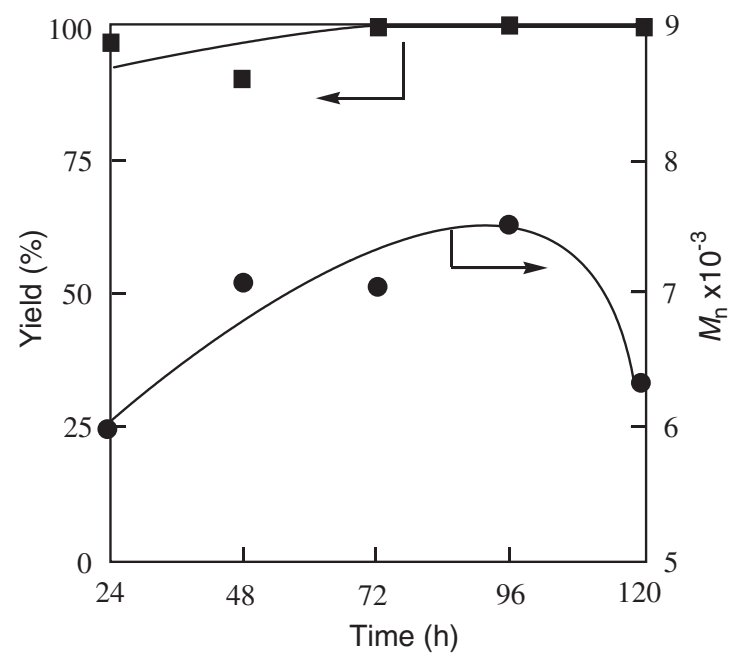

Figure 2. Effect of reaction time on the polyaddition of BEOB with IBDPA. The reaction was carried out with BEOB $(1.0 \mathrm{mmol})$ with IBDPA $(1.0 \mathrm{mmol})$ using TPPB $(5 \mathrm{~mol} \%)$ as

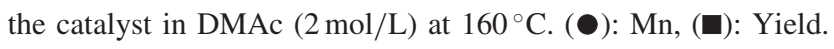

after that, the $M_{\mathrm{n}}$ of the polymers decreased with reaction time. The same tendency was found on the polyaddition of 4,4'-BEOBP with IBDPA under the same reaction conditions. From these results, it seems that the $96 \mathrm{~h}$ is suitable reaction time under this reaction condition, and some decomposition of the ether linkages in the polymer main chain might have occurred as a side reaction for longer time than $96 \mathrm{~h}$.

Based on these results as mentioned above, the polyadditions of certain bis(oxetane)s (BEOB, 1,4BEOMB, 4,4'-BEOBP, 2,2'-BEOBP, TM-BEOBP) with active bis(ester) IBDPA were carried out in the presence of $5 \mathrm{~mol} \%$ of TPPB in DMAc $(2 \mathrm{~mol} / \mathrm{L})$ at $160^{\circ} \mathrm{C}$ for $96 \mathrm{~h}$ as the optimum conditions, affording the corresponding polymers, poly(BEOB-IBDPA), poly(1,4-BEOMB-IBDPA), poly(4,4'-BEOBPIBDPA), poly(2,2'-BEOBP-IBDPA), and poly(TMBEOBP-IBDPA) with $M_{\mathrm{n}} \mathrm{s}$ in the range between $7200-17400$ in $91-99 \%$ yields (Scheme 1). These results and conditions are summarized in Table III. These results show that poly(ether)s with high molecular weights were synthesized by the polyaddition of bis(oxetane) with active bis(ester).

The structures of all obtained polymers were confirmed by the IR, ${ }^{1} \mathrm{H}$ NMR and ${ }^{13} \mathrm{C}$ NMR spectroscopy, and the elemental analysis. This suggests that the polyaddition of bis(oxetane)s with active bis(ester)s proceeded selectively without any side reaction under appropriate reaction conditions.

All the obtained polymers could soluble in common organic solvents, such as DMSO, NMP, DMF, THF, and $\mathrm{CHCl}_{3}$, but insoluble in methanol, ethanol, and $n$-hexane. 
Polyaddition of Bis(oxetane)s with Active Bis(ester)s

Table III. The polyaddition of certain bis(oxetane)s with IBDPA ${ }^{\mathrm{a}}$

\begin{tabular}{cllcrc}
\hline Run no. & Bis(oxetane) & \multicolumn{1}{c}{ Polymer } & $\begin{array}{c}\text { Yield } \\
(\%)^{\mathrm{b}}\end{array}$ & $M_{\mathrm{n}}{ }^{\mathrm{c}}$ & $M_{\mathrm{w}} / M_{\mathrm{n}}$ \\
\hline 1 & BEOB & Poly(BEOB-IPDPA) & 94 & 7200 & 1.67 \\
2 & $1,4-$-BEOMB & Poly(1,4-BEOMB-IPDPA) & 99 & 11200 & 1.88 \\
3 & $4,4^{\prime}$-BEOBP & Poly(4,4'-BEOBP-IPDPA) & 95 & 17400 & 1.78 \\
4 & $2,2^{\prime}$-BEOBP & Poly(2,2'-BEOBP-IPDPA) & 90 & 11400 & 1.69 \\
5 & TM-BEOBP & Poly(TM-BEOBP-IPDPA) & 91 & 12500 & 1.88 \\
\hline
\end{tabular}

${ }^{\mathrm{a}}$ The reaction was carried out with IPDPA $(1.0 \mathrm{mmol})$ with bis(oxetane)s $(1.0 \mathrm{mmol})$ using TPPC $(5 \mathrm{~mol} \%)$ in DMAc $(2 \mathrm{~mol} / \mathrm{L})$ at $160{ }^{\circ} \mathrm{C}$ for $96 \mathrm{~h} .{ }^{\mathrm{b}}$ Insoluble materials in methanol. ${ }^{\mathrm{c}}$ Estimated by SEC (THF) based on polystyrene standards.

\section{CONCLUSIONS}

We demonstrated the synthesis of the novel poly(ether)s by the polyaddition of bis(oxetane)s and active bis(ester)s. The reaction of bis(oxetane)s and active bis(ester)s was examined in the presence of the tetraphenylphosphonium halides as catalysts at $160^{\circ} \mathrm{C}$ for $96 \mathrm{~h}$ in NMP, affording the corresponding poly(ether)s with high molecular weights in satisfactory yields. The obtained polymers could soluble in common organic solvents. Furthermore, an approach to new oxetane resins as application of the reaction of bis(oxetane)s with active bis(ester)s is now under investigation.

\section{REFERENCES}

1. Y. Iwakura and K. Kurita, "Reactive Polymers," Kodansha, Tokyo, 1977.

2. H. Kakiuti, Ed., "Epoxy Resin," Shokodo, Tokyo, 1985.

3. T. Nishikubo, J. Synth. Org. Chem. Jpn., 49, 218 (1991).

4. T. Nishikubo and A. Kameyama, Prog. Polym. Sci., 18, 963 (1993).

5. T. Endo and F. Sanda, Kobunshi, 45, 128 (1996).

6. T. Endo and F. Sanda, Chem. Phys., Macromol. Symp., 107, 237 (1996).

7. E. Takahashi, F. Sanda, and T. Endo, J. Appl. Polym. Sci., 91, 589 (2004).

8. S. Penzek, P. Kubisa, and K, Matyjaszewski, Adv. Polym. Sci., 37, 1 (1980).

9. S. Inoue and T. Aida, in "Ring-Opening Polymerization," K. J. Ivin and T. Saegusa, Ed., Elsevier Applied Science Publishers, London, U.K., 1984, 1, p 185.

10. F. Sanda, T. Kaizuka, A. Sudo, and T. Endo, Macromolecules, 36, 967 (2003).

11. A. S. Pell and G. Pilchere, Trans. Faraday Soc., 61, 71
(1965).

12. T. Kubisa, J. Polym. Sci., Part A: Polym. Chem., 41, 457 (2003).

13. For example, A. Ueyama, M. Mizuno, S. Kanoh, and M. Motoi, Polym. J., 34, 944 (2002).

14. For example, S. Kano, T. Nishimura, Y. Kita, H. Ogawa, M. Motoi, M. Takani, and T. Tanaka, J. Org. Chem., 65, 2253 (2000).

15. For example, H. Ogawa, S. Kanoh, and M. Motoi, Bull. Chem. Soc. Jpn., 70, 1649 (1997).

16. T. Nishikubo, A. Kameyama, and A. Suzuki, React. Funct. Polym., 37, 19 (1998).

17. T. Nishikubo, A. Kameyama, M. Ito, T. Nakajima, and H. Miyazaki, J. Polym. Sci., Part A: Polym. Chem., 37, 2781 (1999).

18. Y. Konno, H. Suzuki, H. Kudo, A. Kameyama, and T. Nishikubo, Polym. J., 36, 114 (2004).

19. T. Nishikubo, A. Kameyama, M. Ito, and A. Suzuki, J. Polym. Sci., Part A: Polym. Chem., 36, 2873 (1998).

20. A. Kameyama, K. Ueda, H. Kudo, and T. Nishikubo, Macromolecules, 35, 3792 (2002).

21. H. Kudo, A. Morita, and T. Nishikubo, Polym. J., 35, 88 (2003).

22. A. Morita, H. Kudo, and T. Nishikubo, Polym. J., 36, 413 (2004).

23. A. Morita, H. Kudo, and T. Nishikubo, J. Polym. Sci., Part A: Polym. Chem., 42, 4570 (2004).

24. A. Kameyama, Y. Yamamoto, and T. Nishikubo, J. Polym. Sci., Part A: Polym. Chem., 31, 1639 (1993).

25. A. Kameyama, Y. Yamamoto, and T. Nishikubo, Macromol. Chem. Phys., 197, 1147 (1996).

26. T. Nishikubo and K. Sato, Chem. Lett., 697 (1991).

27. H. Kudo, T. Kurakata, M. Sasaki, and T. Nishikubo, J. Polym. Sci., Part A: Polym. Chem., 42, 1528 (2004).

28. T. Nishikubo, T. Iizawa, M. Shimojyo, T. Kato, and A. Shiina, J. Org. Chem., 55, 2536 (1990).

29. T. Nishikubo, T. Kato, Y. Sugimoto, M. Tomoi, and S. Ishigaki, Macromolecules, 23, 3406 (1990). 